\title{
Active and passive silica waveguide integration
}

\author{
Hübner, Jörg; Guldberg-Kjær, Søren Andreas
}

Published in:

Proceedings on 27th European Conference on Optical Communication

Link to article, DOI:

10.1109/ECOC.2001.989140

Publication date:

2001

Document Version

Publisher's PDF, also known as Version of record

Link back to DTU Orbit

Citation (APA):

Hübner, J., \& Guldberg-Kjær, S. A. (2001). Active and passive silica waveguide integration. In Proceedings on 27th European Conference on Optical Communication (Vol. 4, pp. 630-631)

https://doi.org/10.1109/ECOC.2001.989140

\section{General rights}

Copyright and moral rights for the publications made accessible in the public portal are retained by the authors and/or other copyright owners and it is a condition of accessing publications that users recognise and abide by the legal requirements associated with these rights.

- Users may download and print one copy of any publication from the public portal for the purpose of private study or research.

- You may not further distribute the material or use it for any profit-making activity or commercial gain

- You may freely distribute the URL identifying the publication in the public portal

If you believe that this document breaches copyright please contact us providing details, and we will remove access to the work immediately and investigate your claim. 


\title{
Active and Passive Silica Waveguide Integration
}

\author{
Jörg Hübner and Søren Guldberg-Kjær \\ COM \\ Technical University of Denmark, Ørsteds Plads, Byg. 345v \\ Dk-2800 Kgs. Lyngby, Denmark \\ Email: johu@com.dtu.dk
}

Abstract: Integrated optical amplifiers are currently regaining interest. Stand-alone single integrated amplifiers offer only limited advantage over current erbium doped fiber amplifiers, whereas arrays of integrated amplifiers are very attractive due to miniaturization and the possibility of mass production. The increasing complexity and functionality of optical networks prompts a demand for highly integrated optical circuits. On-board optical amplifiers, monolithically integrated with functionalities like switching or multiplexing/demultiplexing will allow flexible incorporation of optical integrated circuits in existing and future networks without affecting the power budget of the system. Silica on silicon technology offers a unique possibility to selectively dope sections of the integrated circuit with erbium where amplification is desired. Some techniques for active/passive integration are briefly reviewed and a silica on silicon based approach is discussed in more detail.

\section{Introduction}

Based on the success of erbium doped fiber amplifiers (EDFA), a lot of research effort was dedicated to erbium doped integrated optical amplifiers during the early 90 's. The goal at that time was to replace the EDFA by a miniaturized, mass-producable and thereby cost effective integrated amplifier. After a few years of intense research, it became obvious that the excellent specifications of EDFA's are out reach for an integrated component since ultra compact traveling wave amplifiers require very high doping levels. Effects like upconversion and clustering of erbium ions decrease the efficiency of high doping levels, which is the main reason why it is rather unlikely that high performance EDFA will be replaced by their integrated counterparts. During the mid 90's people argued that a coiled erbium doped fiber would always be superior to any planar erbium doped amplifier. As a result of these considerations the interest in erbium doped planar amplifiers (EDPA) decreased significantly.

What seemed a reasonable statement at that time is currently being reconsidered. The main reason for this is the introduction of wavelength division multiplexing (WDM), allowing all optical networks of advanced topologies. Indeed there was no need for an EDPA's in the early point to point single wavelength transmission links of the early nineties. Basically, there was no need for integrated optics in these early transmission links. The need for integrated optics was triggered by the development of WDM and dense-WDM (DWDM) systems. Especially alloptical DWDM ring networks require advanced functionalities in large quantities that can only be provided by integrated optics. The arrayed waveguide grating (AWG) as a largely scalable multiplexer/demultiplexer is clearly a key component in these networks. Although AWGs are produced using several technologies, it is silica on silicon based circuits that dominate the market and show best performance.

A next and natural step in the development of integrated optics is the addition of an amplifying section to existing components e.g. AWGs. This allows loss-less components or even components with a small booster-or pre-amplifier to compensate for short link transmission losses, and allows functionalities like for example an active gain equalizer consisting of two AWG's and an amplifier array.
In advanced network topologies, some channels will pass through more components than others and hence suffer considerably higher transmission loss. Complicated power budget plans for re-routable networks have to be adapted and reconsidered continuously with network growth. Lossless components offer the flexibility that they can be placed in the network without affecting the power budget. With growing size and complexity of all-optical networks, loss less components and adjustable channel equalizers will become increasingly important.

More and more specialized functionality will be required and we will see a trend towards application specific integrated optical circuits, containing passive functionality like multiplexers/demultiplexers or channel interleavers integrated together with channel monitors, amplifier arrays, variable optical attenuators etc.

In these cases the planar amplifier does not compete with the fiber amplifier any more, it is an integral part of an integrated optical circuit, delivering advanced functionality. It merely supplements the high performance $\mathrm{C}$ and $\mathrm{L}$ band EDFA, which is and will be an essential part of all-optical networks.

\section{Integration}

A requirement for fabrication of such components is the successful integration of active and passive areas on the wafer, where the respective active (amplifying) section and passive (e.g. multiplexing) circuitry is defined. Success criteria include low transition loss from active to passive waveguides and, even more important, extremely low back reflection into the active waveguide section. Another very important criteria is manufacturability, meaning that fabricating components with active and passive integration should have a yield comparable to the yield of the single components (active or passive) alone. Low cost mass production of components containing EDPA arrays includes hybridization of semiconductor pump lasers, a technology, which is well under way but beyond the scope of this paper [1]

In the following a brief review of possibilities of active and passive integration is given, followed by a more detailed description of the work in silica on silicon done at COM.

A rather straight forward but technologically difficult approach is the mounting of semiconductor optical 
amplifiers (SOA) on a silica-on-silicon planar lightwave circuit [1]. This transfers all advantages and disadvantages of the extremely compact SOA to silica planar lightwave circuits. Especially the small mode field diameter of the SOA and the refractive index mismatch between the semiconductor amplifier and the glass waveguide resulting in loss and possible feedback due to back-reflections require careful engineering. Due to strong non-linearities hybrid mounted SOAs are mostly suited for single channel amplification.

A technology which is very well suited for integration of active and passive functionality has been demonstrated by Van den Hooven et al. [2]. The waveguide material consisting of alumina constitutes an excellent host for erbium ions. The erbium doping itself is done by ion implantation so parts of the waveguide structure can be selectively doped allowing seamless integration of active and passive waveguide sections

Phosphate glass based integrated amplifiers with waveguides defined by ion exchange show a very high gain per unit length and are commercially available [3]. As the standard phosphate glass based substrate is uniformly doped there are two main approaches to integrated passive and active functionality. The first approach consists of having a doped and a non-doped substrate with the respective active and passive circuitry defined in separate lithographic steps. These two parts can then be glued or fused together. The second approach requires a wafer substrate consisting of one or more doped and non-doped areas. Lithography is carried out on this wafer thereby defining active and passive circuitry in one step. This ensures perfect lateral alignment of passive and active regions.

A similar approach is taken with the silica-on-silicon technology at COM [4] (another approach using silica-onsilicon is reported in [5]). The erbium doped (active) as well as the passive layer are deposited by plasma enhanced chemical vapor deposition (PECVD). First the germanium doped passive core glass layer is deposited uniformly over thermally grown oxide buffer layer. Using lithography and reactive ion etch (RIE), areas of this core layer are removed. The etch process is optimized to produce sloped sidewalls. Next the active core layer is deposited with a layer thickness identical to the passive core layer. The wafer contains now areas with the passive core layer and areas with the active core layer on top of the passive core layer. Using a mask, which is the negative of the mask used to etch the passive core layer, the active core layer is now removed from the areas where it covers the passive layer. The result after this etch step consists of a planar core layer with areas of passive and active glass. The waveguide circuitry, passive and active, is now defined in one lithography step. This ensures perfect lateral alignment. The horizontal alignment is dependent on the etch precision which is in our case typically better than $100 \mathrm{~nm}$. Figure 1 shows a scanning electron microscope (SEM) picture of the interface between a passive and active section of the waveguide. Using this technology transition losses are measured to be below $0.03 \mathrm{~dB} /$ transition and backreflections are suppressed more than $70 \mathrm{~dB}$ (see figure 2 ).

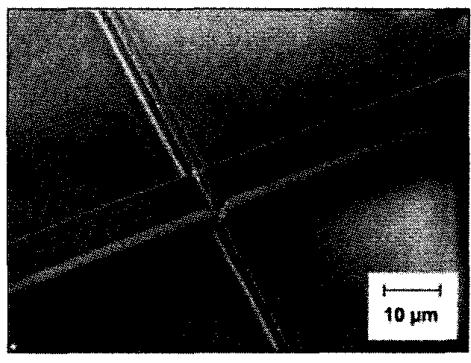

Figure 1: SEM picture of the boundary between active and passive region (picture courtesy of Christian Laurent-Lund).

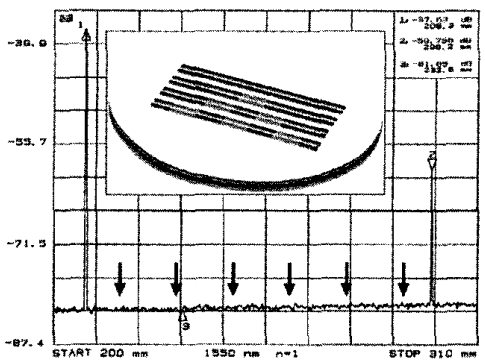

Figure 2: Reflection measurement for a waveguide with three erbium doped sections. The time domain reflectometer shows the reflection of the end facets of the waveguides and no reflections from the section transitions. The inset shows the layout of the waveguides with active and passive sections.

\section{Conclusion}

The interest for erbium doped integrated planar amplifiers is currently increasing. This interest is founded in the need of low cost medium performance amplifier arrays. Planar amplifiers are not supposed to replace EDFA's but rather supplement them where less performance and a higher number of amplifiers (e.g. single channel amplification) is needed. A major step towards lower cost and high manufacturability is the on-board integration of amplifier arrays together with other functionalities like multiplex/demultiplexing. The possibility to integrate a large variety of passive functionality with amplifying sections allows loss-less application-specific integrated optical circuits as building blocks for future high performance all-optical networks.

\section{References}

11 K. Kato et al., IEEE J. Selected Topics in Quant. Electr., 2000, 6(1), pp. 4-13.

12/ G.N. Van den Hoven et al., Appl. Phys. Lett. 1996, 68 (14), pp. 1886-1888.

13/ Y.Jaouen et al., PTL 1999, Vol. 11, No. 9, pp.11051107.

14/ Chr. Laurent-Lund et al. IEEE Photon. Technol. Lett. ,1998, 10, (10), pp. 1431-1433.

15/ M. Ogumaet al., OAA, 1996,(OSA), pp. $48-51$. 